\title{
Reproduction of a Protocell by Replication of a Minority Molecule in a Catalytic Reaction Network
}

\author{
Atsushi Kamimura ${ }^{1, *}$ and Kunihiko Kaneko ${ }^{2,3}$ \\ ${ }^{1}$ Department of Applied Physics, The University of Tokyo, 7-3-1, Hongo, Bunkyo-ku, Tokyo 113-8656, Japan \\ ${ }^{2}$ Department of Basic Science, The University of Tokyo, \\ 3-8-1, Komaba, Meguro-ku, Tokyo 153-8902, Japan \\ ${ }^{3}$ Complex Systems Biology Project, ERATO, JST, Tokyo, Japan
}

(Received 30 April 2010; published 29 December 2010)

\begin{abstract}
For understanding the origin of life, it is essential to explain the development of a compartmentalized structure, which undergoes growth and division, from a set of chemical reactions. In this study, a hypercycle with two chemicals that mutually catalyze each other is considered in order to show that the reproduction of a protocell with a growth-division process naturally occurs when the replication speed of one chemical is considerably slower than that of the other chemical, and molecules are crowded as a result of replication. It is observed that the protocell divides after a minority molecule is replicated at a slow synthesis rate, and thus, a synchrony between the reproduction of a cell and molecule replication is achieved. The robustness of such protocells against the invasion of parasitic molecules is also demonstrated.
\end{abstract}

DOI: 10.1103/PhysRevLett.105.268103

PACS numbers: 87.17. $-\mathrm{d}, 05.40 .-\mathrm{a}, 82.39 .-\mathrm{k}$

Filling a gap between just a set of catalytic reactions and a reproducing cell is a difficult problem; however, it is essential to unveil the origin of life, to establish a theory for a living state, and to experimentally synthesize protocells [1]. Several theoretical attempts have been made [2-4]. For reproduction of cells, it is necessary to replicate functional polymers. At a primitive stage of life, however, errors in the sequence of polymers are inevitable during their replications, which prevent the transfer of information through replications, as known as an error catastrophe [4]. To resolve this error-catastrophe problem, a hypercycle was proposed, in which different molecular species mutually catalyzed the replication of each other. This hypercycle, however, introduced another problem, i.e., the emergence of parasitic molecules that are catalyzed by other molecules but do not in turn catalyze other molecules. Because such parasitic molecules are in a majority, the reproduction of mutually catalytic molecules cannot be sustained.

One possible way to resolve the problem of parasitism is to use compartmentalization [4-6]. When molecules are compartmentalized in a protocell, the unit of selection is shifted from a molecule to a protocell. Then, the protocells dominated by parasites are selected out, leaving behind protocells without parasites. However, such a compartmentalization introduces further complexity to the primitive form of life. Then, other membrane molecules would be needed, which also need to be replicated. To avoid the further introduction of complexity, it is important to develop a simple mechanism for compartmentalization of a protocell.

Another important issue associated with protocells is the origin of heredity, i.e., the existence of specific molecules (such as DNA in the present cell) that are well preserved in the offspring cells and also control the behavior of the cells. By considering a simple hypercycle with only two chemicals, we have previously shown that the minority chemical with a slower synthesis rate plays the role of a heredity-carrier molecule. In fact, the relevance of such minority molecules in the hypercycle for evolvability and for the elimination of parasites is demonstrated [7,8]. Here again, reaction in a compartment and the division of such a protocell was assumed.

Here, we address the following questions. Can we explain the origin of a protocell with compartmentalization and its growth-division cycle, in terms of a reactiondiffusion process with minority molecules? How does the division process of protocells maintain synchrony with the replication of such minority molecules? We address these questions by simply introducing catalytic reactions which can induce crowdedness in molecules as a result of replication. We use the physicists' golden rule to study one the most simplest systems, i.e., a system consisting of only two chemical species that mutually catalyze the replication of each other, with the Brownian motion of molecules having simple exclusion volumes. From the results of a stochastic simulation, we show that both of the difference between the synthesis speed of the two molecular species and the crowding effect of molecules lead not only to the formation of compartments as a cluster of such molecules but also to their recursive division after the replication of a minority molecule, even though no complicated mechanisms are assumed. Crowdedness of molecules around the minority molecule leads to compartmentalization, whereas infrequent replication of such a minority molecule induces the division of a compartment. Therefore, the reproduction of a protocell, which is essentially a steady cluster of molecules, progresses in synchrony with the replication of a minority molecule. We also demonstrate that this reproduction process tenaciously resists parasites. 
We consider two molecular species that mutually catalyze the replication of each other. The reaction processes are expressed as follows:

$$
\begin{array}{cc}
X+Y \stackrel{p \gamma_{X}}{\longrightarrow} 2 X+Y, \quad X+Y \stackrel{p \gamma_{Y}}{\longrightarrow} 2 Y+X, \\
X \stackrel{a_{X}}{\rightarrow}, \quad Y \stackrel{a_{Y}}{\longrightarrow} 0 .
\end{array}
$$

The first process represents mutual catalytic replication with rate $p$, where the synthesis fractions of $X$ and $Y$ are $\gamma_{X}$ and $\gamma_{Y}$, respectively. The second process represents the natural decomposition of $X$ and $Y$ with rates $a_{X}$ and $a_{Y}$, respectively.

Because it is necessary to ensure discreteness in a molecular number, we adopt stochastic simulations rather than reaction-diffusion equations. In fact, the importance of ensuring discreteness in the molecular number has been discussed [9,10], while crowdedness in molecules is taken into account here, which lead to a drastic change to the behavior of the system. We assume that both $X$ and $Y$ molecules have a spherical shape with the same diameter $(\sigma)$. These molecules are confined to a box with dimensions $L_{x} \times L_{y} \times L_{z}$, with elastic boundaries. Further, these molecules have the same mass and diffusion constant $(D)$. Their motions obey the overdamped Langevin equations, which are numerically integrated by the Ermak-McCammon algorithm [11], such that $\Delta x=-\frac{D \Delta t}{k_{B} T} \nabla U(r)+S(t)$. Here, $S(t)$ is a Gaussian white noise such that $\langle S(t)\rangle=0,\left\langle S(t) S\left(t^{\prime}\right)\right\rangle=$ $2 D \Delta t \delta\left(t-t^{\prime}\right)$, with temperature $k_{B} T=1$, where $k_{B}$ denotes the Boltzmann constant. In the simulation, we set $\Delta t=0.00005$. The interaction potential $U(r)$ between molecules is a Hertzian potential, $\phi(r)=E \mid \sigma-$ $\left.r\right|^{5 / 2} \Theta(\sigma-r)$, where $E=10000 \epsilon / \sigma^{5 / 2}$ with an energy unit $\epsilon$, and the characteristic length scale $\sigma$ (i.e., the diameter) of molecules and $\Theta$ is a Heaviside step function [12]. We start our simulation with the initial condition in which a single molecule $Y$ is surrounded by $X$ molecules [13].

At each time step, if the distance between every pair of $X$ and $Y$ is shorter than $\sigma$, the replication reaction occurs with a probability $p^{\prime}=p \Delta t$. If the reaction occurs, a new molecule $X$ or $Y$ is added to the system, with probability $\gamma_{X}$ or $\gamma_{Y}$, respectively. Hence, the reaction rate for the replication of $X$ and $Y$ for a single reaction pair is given by $r_{X} \equiv p \gamma_{X}$ and $r_{Y} \equiv p \gamma_{Y}$, respectively. The position of the new molecule is chosen as $(x, y, z)=\left(x_{0}+f_{x}, y_{0}+\right.$ $\left.f_{y}, z_{0}+f_{z}\right)$, where $\left(x_{0}, y_{0}, z_{0}\right)$ is the center between the reaction pair and $\left(f_{x}, f_{y}, f_{z}\right)$, which is randomly chosen from $-\sigma<f_{x}, f_{y}, f_{z}<\sigma$, by also avoiding overlap with the reaction pair. The decomposition process is carried out to remove all $X(Y)$ molecules, with a probability $a_{X}^{\prime}=$ $a_{X} \Delta t \quad\left(a_{Y}^{\prime}=a_{Y} \Delta t\right)$ at each step. Here, we fix the parameters as $\sigma=1, D=1$, and $p=10^{3}$ and study the behavior of the system against the changes in the independent parameters $\gamma_{Y}, a_{X}$, and $a_{Y}$, while $\gamma_{X}$ is set as $\gamma_{X}=1-\gamma_{Y} \geq \gamma_{Y}$.
First, we examine the formation of a cluster by considering a single $Y$ molecule and suppressing its replication and decomposition (i.e., $\gamma_{Y}=a_{Y}=0$ ). If $a_{X}$ is large, none of the $X$ molecules remain to exist, whereas a stationary cluster of $X$ molecules is formed around the single $Y$ molecule when $a_{X}$ is sufficiently small. For it, we fix $a_{X}=4$ in the following. Indeed, we have computed the density distribution function $N_{X}$ of $X$ as a function of distance $r$ from the single $Y$. The tail of the function for a large $r$ is fitted well by the isotropic steady-state solution of the diffusion equation $N_{X} \sim \exp \left(-r / r_{0}\right) / r$ with $r_{0}=\sqrt{D / a_{X}}$. For a small $r$, the value of the function is suppressed without $1 / r$ divergence owing to crowded molecules. Under the crowded condition, $X$ and $Y$ continuously overlap each other in the replication reaction. Then, the replication rate of $X$ by a single $Y$ molecule, denoted as $R_{X}$, is given by $R_{X}=r_{X} \bar{n}_{X}$, where $\bar{n}_{X}$ denotes the average number of $X$ molecules that overlap with the single $Y$ molecule. In the present simulations, $\bar{n}_{X} \sim 2$ [14]. Under the present crowded condition, the number of $X$ molecules, $N_{C X}$, in a steady cluster is estimated by the balance between the replication and decomposition of $X$ molecules, as $R_{X}=a_{X} N_{C X}$. By using $\bar{n}_{X} \sim 2$, we then estimate $N_{C X}$ as $\sim 500$, which is consistent with numerical results.

Now, we consider the replication of $Y$ molecules with a rate that is slower than that of $X$. First, in Fig. 1, we show an example of the growth-division process of a cluster and provide the condition for it later. This process occurs as follows. For most of the time, the cluster maintains itself by replicating the $X$ molecules, whereas the replication of the $Y$ molecule rarely occurs. Once the $Y$ molecule replicates, the distance between the two $Y$ molecules is increased by diffusion, and these molecules contribute to the replication of $X$ molecules. As the two $Y$ molecules gradually move away from each other, the spatial distribution of $X$, synthesized by each $Y$, starts to elongate in the direction of
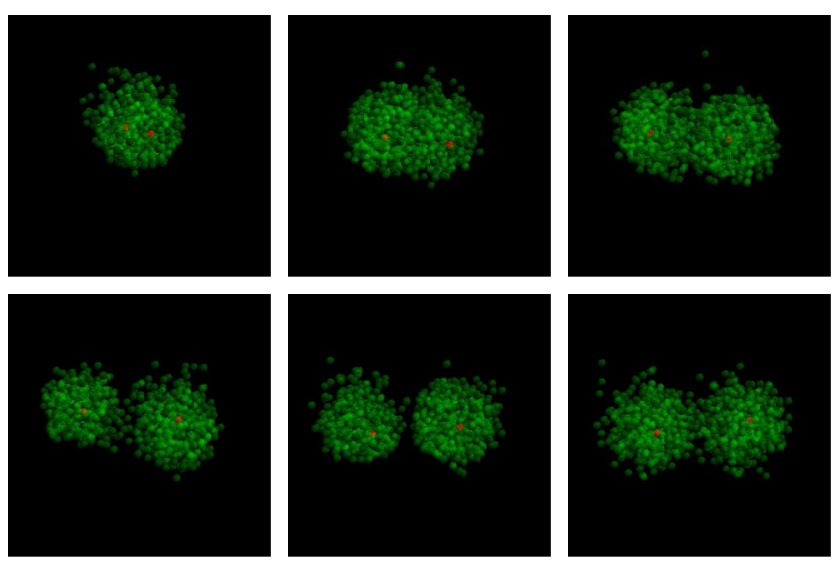

FIG. 1 (color online). Snapshots after the replication of $Y$. The green semitransparent particles represent the $X$ molecules. The red particles represent the $Y$ molecules, located deep within the clusters. Time evolves from the left top to right bottom, as $t=0.1,0.5,1,1.5,2,2.5$. The parameter values are $r_{Y}=0.01$, $a_{X}=4$, and $a_{Y}=0.002$. 
the two $Y$ molecules. Since the decomposition of the $X$ molecule is dominant because it is located away from a $Y$ molecule, the cluster of $X$ molecules forms a dumbbell shape, and it finally splits into two (see also movies in the supplementary material [15]).

In fact, for this growth-division cycle to repeat, there is a restriction on the values of several parameters. First, the replication of $Y$ should be sufficiently slow. After the replication of a new $Y$ molecule, it takes some time for the two $Y$ molecules to be separated at a distance of the size of the cluster. If another replication of $Y$ occurs during this time, the cluster of $X$ molecules cannot split into two because the replication of $X$ molecules by the third $Y$ molecule already begins. Second, the decay rate of $Y$ should be sufficiently low. If this is not the case, the $Y$ molecules would completely disappear before the division of a cluster. On the other hand, it should be noted that the cluster splits by the decomposition of $X$ so that its rate $a_{X}$ is higher than $a_{Y}$.

By taking several parameter values for $\gamma_{Y}$ and $a_{Y}$, we have plotted the time evolution of the total number of molecules by starting from a single- $Y$ initial condition in Fig. 2. We have found the following three typical behaviors: extinction, division, and explosion. In the extinction phase, all the molecules decay, and finally no molecules remain. In the parameters shown in this figure, extinction is initiated by the decomposition of a single $Y$ molecule, followed by the extinction of $X$ molecules. Here, first, the number of molecules fluctuates at around a constant value, corresponding to a single cluster, and then, it suddenly decreases with the disappearance of the $Y$ molecule. (When $a_{X}$ is considerably larger than $a_{Y}$, the extinction of $X$ molecules precedes that of $Y$.)

In the division behavior, a stepwise increase in the total number of molecules is observed. For reference, we also plot dotted lines in Fig. 2 at the level of several multiples of the number of molecules in a single cluster. The total

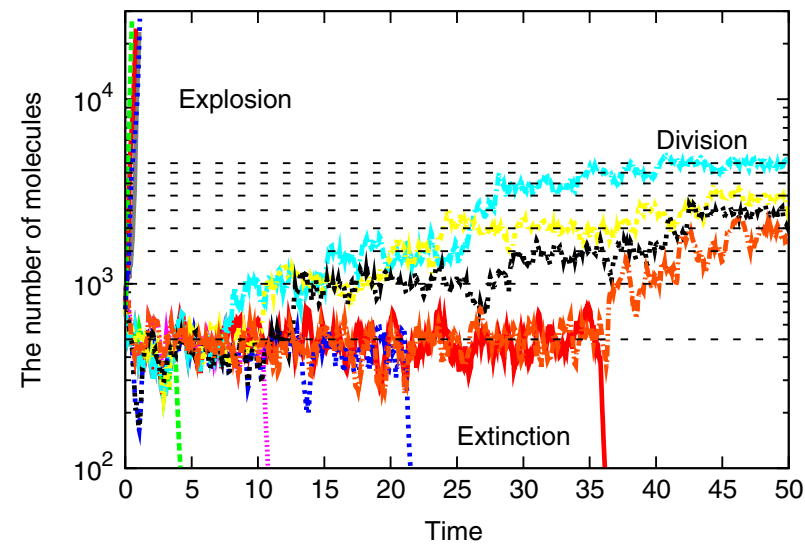

FIG. 2 (color online). Time evolution of the total number of molecules. Data are obtained from the following three sets of parameters with several samples; $r_{Y}=0.001, a_{Y}=0.2$ (leading to extinction), $r_{Y}=0.01, a_{Y}=0.002$ (division), and $r_{Y}=1$, $a_{Y}=0.02$ (explosion). Here, we set $a_{X}=4$. The dotted lines represent several multiples of the molecule number of a single cluster, $N_{C X}$. number of molecules fluctuates around one of the dotted lines and gradually increases, as is consistent with the division process of a certain size of a cluster. In the explosion case, the number of molecules show an exponential increase without a steplike behavior. The number of molecules increases by maintaining a single cluster. Typical snapshots of the division and explosion cases are shown in Fig. 3, where the green particles represent the $X$ molecules.

In Fig. 3, a phase diagram of these three behaviors is plotted on the $r_{Y}-a_{Y}$ plane by fixing the decay rate of $X$ at $a_{X}=4$. The division phase lies at smaller $r_{Y}$ and $a_{Y}$. An increase in $a_{Y}$ leads to extinction, whereas an increase in $r_{Y}$ leads to explosion. Near the boundary between the division and the extinction phases, the two behaviors coexist.

The boundaries between the phases are estimated as follows. In the case of the boundary of the extinction phase, we note the balance between the replication and the decay of $Y$. The replication rate of a $Y$ molecule in the cluster is estimated by $\sim R_{Y}=r_{Y} \bar{n}_{X}$, and the decay rate is estimated by $\sim a_{Y}$. In the case of the division phase, the replication rate of $Y$ should be greater than its decay rate so that the region satisfies

$$
a_{Y}<R_{Y}
$$

This boundary is plotted in the $r_{Y}-a_{Y}$ plane, which is consistent with our simulation results.

The boundary between the division and the explosion phases is estimated by the balance between the replication rate of $Y$ and the size of the cluster. The linear size of a single cluster, $L_{C}$, is estimated by $L_{C} \sim N_{C X}^{1 / 3}$. The division process starts by the replication of $Y$ and is completed

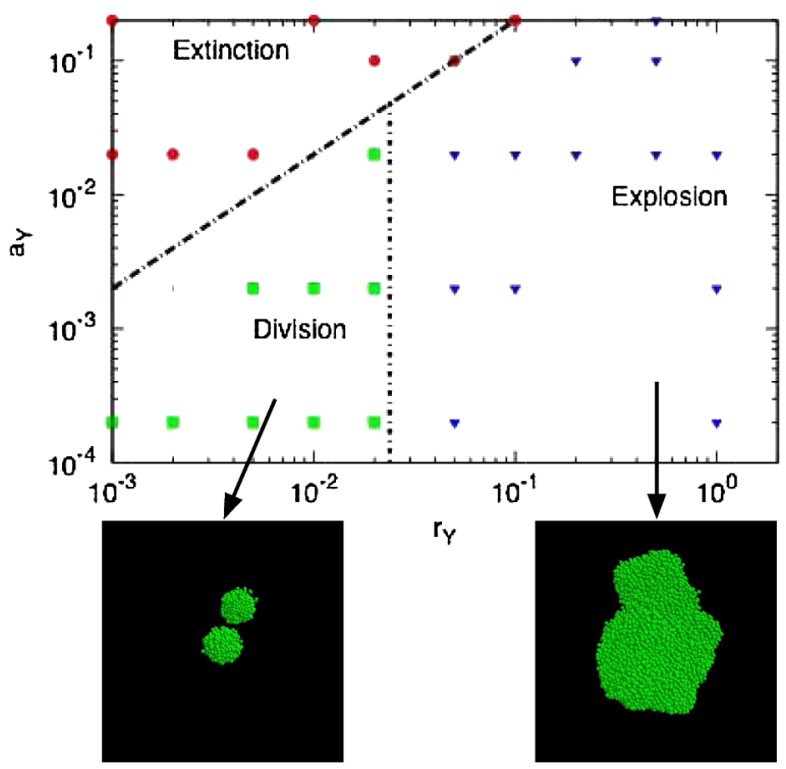

FIG. 3 (color online). Phase diagram of our model in $r_{Y}-a_{Y}$ space. The parameters are $p=10^{3}$ and $a_{X}=4$. The plane is divided into the following three regions: extinction $(\bigcirc)$, division $(\square)$, and explosion $(\nabla)$. At the parameters with + points, two behaviors of the adjacent regions coexist. We also plot the theoretically estimated boundaries given by Eqs. (3) and (4). 
when the two $Y$ molecules diffuse approximately to the distance $L_{C}$. We denote the typical time scale of dividing processes by $\tau_{D}$, and we obtain $L_{C}^{2} \sim D_{Y} \tau_{D}$, where $D_{Y}$ denotes the effective diffusion constant of $Y$. Here, $D_{Y}$ is slightly increased from $D=1$ because of the repulsive interactions with $X$ molecules, and it is estimated as $D_{Y} \sim 3$ from the simulations for Fig. 3..

At the phase boundary, only a single replication of $Y$ should occur during this time $\tau_{D}$ to complete the division, so that $R_{Y} \tau_{D}=1$. Here, $\tau_{D}=L_{C}^{2} / D_{Y}=\left(R_{X} / a_{X}\right)^{2 / 3} / D_{Y}$. Thus, the boundary is expressed as

$$
R_{Y}=D_{Y}\left(\frac{a_{X}}{R_{X}}\right)^{2 / 3}
$$

The estimated boundary is plotted in Fig. 3, which is also consistent with our results.

In summary, we have studied a simple reaction system of two mutually catalytic molecules that diffuse under a crowded condition. When the replication speed considerably differs between the two molecular species, the molecule with a slower replication rate becomes a minority molecule, which leads to the formation of a steady cluster of molecules, while the separation of a pair of the replicated minority molecule under crowded condition leads to the division of the cluster of molecules into two. Hence, the division phase is a consequence of synergetic effect between minority molecules and the crowdedness. Here, we have presented the simulation results for a three-dimensional case; however, we have confirmed the existence of the present three phases, in particular, the division of a protocell in a two dimensional case.

To further examine whether the present protocell resists parasitic molecules, we have also extended our model to include parasitic molecules $X^{\prime}$ and $Y^{\prime}$, which are catalyzed by other molecular species but cannot catalyze the replication of other molecules. If the variety and/or replication rates of the parasites are large, they would dominate, leading to the growth suppression of protocells dominated by them (see supplementary material [15]). In particular, when parasite $Y^{\prime}$ appears, it is spontaneously emitted from the cluster, so that $Y^{\prime}$ parasites are eliminated.

It should be noted that the reaction scheme given in Eq. (1) is extremely simplified. For such reactions to progress, some resources are necessary for the synthesis of $X$ and $Y$. If such resources are sufficiently supplied, the original model [Eq. (1)] is derived. In reality, however, these resources have to be supplied from the outside [16]. As the size of the cluster increases, their supply cannot be penetrated deeper into the cluster; therefore, replication is limited only at the periphery, whereas decomposition occurs in a bulk. Hence, the growth of a cluster without division stops when the cluster achieves a certain size. In contrast, when the cluster divides into two before it reaches the size threshold, the molecules continue to replicate.

In conclusion, the formation of a compartment and its growth-division cycle are shown to be a natural outcome of mutually catalytic reactions with a minority molecule species. As already discussed, the minority molecule $Y$ can act as a carrier of heredity, as it is preserved well, controls the characteristic of the protocells, and gives evolvability $[7,8,17]$. Here, we have shown that the replication of this minority molecule under crowded condition is synchronized with the division of a protocell. These characteristics of the minority molecule $Y$ agree well with those required by genetic information, played by DNA in the present cell. The question about the origin of genetic information from catalytic reactions, addressed by Dyson [2], is thus answered.

This work is supported in part by the Japan Society for the Promotion of Science.

*Present address: Institute of Industrial Science, The University of Tokyo, 4-6-1, Komaba, Meguro-ku, Tokyo 153-8505, Japan.

[1] Protocells Bridging Nonliving and Living Matter, edited by S. Rasmussen et al. (MIT Press, Cambridge, MA, 2009).

[2] F. Dyson, Origins of Life (Cambridge University Press, Cambridge, England, 1985).

[3] S. A. Kauffman, J. Theor. Biol. 119, 1 (1986); S. Krishina and S. Jain, Proc. Natl. Acad. Sci. U.S.A. 99, 2055 (2002); D. Segre, D. Ben-Eli, and D. Lancet, Proc. Natl. Acad. Sci. U.S.A. 97, 4112 (2000); C. Furusawa and K. Kaneko, Phys. Rev. Lett. 90, 088102 (2003).

[4] M. Eigen and P. Schuster, The Hypercycle (Springer, New York, 1979); M. Eigen, Steps Towards Life (Oxford Univ. Press, Oxford, 1992).

[5] J. Maynard-Smith, Nature (London) 280, 445 (1979); E. Szathmary and L. Demeter, J. Theor. Biol. 128, 463 (1987).

[6] S. Altmeyer and J. S. McCaskill, Phys. Rev. Lett. 86, 5819 (2001); M. Boerlijst and P. Hogeweg, Physica (Amsterdam) 48D, 17 (1991).

[7] K. Kaneko and T. Yomo, J. Theor. Biol. 214, 563 (2002).

[8] K. Kaneko, Phys. Rev. E 68, 031909 (2003); Adv. Chem. Phys. 130, 543 (2005).

[9] N. M. Shnerb et al., Proc. Natl. Acad. Sci. U.S.A. 97, 10322 (2000).

[10] Y. Togashi and K. Kaneko, Phys. Rev. Lett. 86, 2459 (2001); Physica (Amsterdam) 205D, 87 (2005).

[11] D. L. Ermak and J. A. McCammon, J. Chem. Phys. 69, 1352 (1978).

[12] Specific form is not important. Consideration of finite molecular size is essential for the crowding effect.

[13] As long as there are $X$ and $Y$ molecules, the same behavior is exhibited after some time.

[14] This value depends on $D$ and $p ; \bar{n}_{X}$ can be roughly written as $p \sigma^{2} / D$, whereas with a decrease in $D$ it saturates at $\sim 12$, the value at the hexagonal close packing.

[15] See supplementary material at http://link.aps.org/ supplemental/10.1103/PhysRevLett.105.268103.

[16] A. Kamimura, S. Yukawa, and N. Ito, J. Phys. Soc. Jpn. 74, 1071 (2005); 75, 024005 (2006).

[17] T. Matsuura et al., Proc. Natl. Acad. Sci. U.S.A. 99, 7514 (2002). 